University of Wollongong

Research Online

Faculty of Engineering and Information

Faculty of Engineering and Information

Sciences - Papers: Part A

Sciences

$1-1-2014$

Semi-analytical solutions for the reversible Selkov model with feedback

delay

Khaled. Al Noufaey

University of Wollongong, ksnan708@uowmail.edu.au

Timothy Marchant

University of Wollongong, tim@uow.edu.au

Follow this and additional works at: https://ro.uow.edu.au/eispapers

Part of the Engineering Commons, and the Science and Technology Studies Commons

Research Online is the open access institutional repository for the University of Wollongong. For further information contact the UOW Library: research-pubs@uow.edu.au 


\title{
Semi-analytical solutions for the reversible Selkov model with feedback delay
}

\begin{abstract}
Semi-analytical solutions for the reversible Selkov, or glycolytic oscillations model, are considered. The model is coupled with feedback at the boundary and considered in one-dimensional reaction-diffusion cell. This experimentally feasible scenario is analogous to feedback scenarios in a continuously stirred tank reactor. The Galerkin method is applied, which approximates the spatial structure of both the reactant and autocatalyst concentrations. This approach is used to obtain a lower-order, ordinary differential equation model for the system of governing equations. Steady-state solutions, bifurcation diagrams and the region of parameter space, in which Hopf bifurcations occur, are all found. The effect of feedback strength and delay response on the parameter region in which oscillatory solutions occur, is examined. It is found that varying the strength of the feedback response can stabilize or destabilize the system while increasing the delay response usually destabilizes the reaction-diffusion cell. The semianalytical model is shown to be highly accurate, in comparison with numerical solutions of the governing equations.
\end{abstract}

\section{Keywords}

model, selkov, reversible, solutions, analytical, delay, semi, feedback

Disciplines

Engineering | Science and Technology Studies

\section{Publication Details}

Al Noufaey, K. \& Marchant, T. R. (2014). Semi-analytical solutions for the reversible Selkov model with feedback delay. Applied Mathematics and Computation, 232 (2014), 49-59. 


\title{
Semi-analytical solutions for the reversible Selkov model with feedback delay
}

\author{
K. S. Al Noufaey and T. R. Marchant, \\ School of Mathematics and Applied Statistics, \\ The University of Wollongong, \\ Wollongong, 2522, N.S.W., \\ Australia.
}

January 7, 2014

\begin{abstract}
Semi-analytical solutions for the reversible Selkov, or glycolytic oscillations model, are considered. The model is coupled with feedback at the boundary and considered in onedimensional reaction-diffusion cell. This experimentally feasible scenario is analogous to feedback scenarios in a continuously stirred tank reactor. The Galerkin method is applied, which approximates the spatial structure of both the reactant and autocatalyst concentrations. This approach is used to obtain a lower-order, ordinary differential equation model for the system of governing equations. Steady-state solutions, bifurcation diagrams and the region of parameter space, in which Hopf bifurcations occur, are all found. The effect of feedback strength and delay response on the parameter region in which oscillatory solutions occur, is examined. It is found that varying the strength of the feedback response can stabilize or destabilize the system while increasing the delay response usually destabilizes the reaction-diffusion cell. The semi-analytical model is shown to be highly accurate, in comparison with numerical solutions of the governing equations.
\end{abstract}

Keywords: semi-analytical solutions; reaction-diffusion equations; feedback delay; reversible Selkov model; Hopf bifurcations.

\section{Introduction}

Some chemical reactions, such as the Bray-Liebhafsky, Belousov-Zhabotinsky and BriggsRausher systems, undergo periodic variations in concentrations which can be visualized via changes in colour; see [1] for a review of these systems and other oscillatory phenomena. These reactions have helped to motivate great interest, by both theoreticians and experimentalists, in the study of oscillatory chemical systems. The most common chemical reactor scenario is the continuous flow well-stirred tank reactor (CSTR). Usually, a system of ordinary differential equations (odes) governs a CSTR, which can be analyzed by standard techniques. However another important reactor scenario is the reaction-diffusion cell, which is governed by a system of partial differential equations (pdes), and is not as easy to analyze.

[2] considered a simple mathematical model of a glycolytic system, which is based on Selkov's model [3], which exhibits periodic oscillations. This scheme, known as the reversible 
Selkov model [4], is

$$
A \rightleftharpoons S, \quad S+2 P \rightleftharpoons 3 P, \quad P \rightleftharpoons B,
$$

where $\mathrm{S}$ (the reactant) and $\mathrm{P}$ (the autocatalyst) are the concentrations of adenosine triphosphate (ATP) and adenosine diphosphate (ADP), respectively. ATP is a high energy molecule with three phosphate groups that a cell uses to extract and store energy from other molecules such as carbohydrates. ADP is a low-energy molecule that has one phosphate group less than the ATP molecule. A and B represent controllable source and sinks, respectively; Glucose or fructose are examples of the source inputs into this system.

[2] analyzed the reversible Selkov reaction (1) in a CSTR and found two types of steadystate bifurcation diagrams, the unique and the breaking-wave patterns. In addition, they found the region where Hopf bifurcations occur and discussed their stability. [4] analyzed the symmetry-breaking instability in (1) and found the time periodic oscillations of the homogeneous model are subject to a symmetry-breaking instability, via Hopf-bifurcation, by coupling with molecular diffusion in a distributed system. This instability generates sinusoidal heterogeneous waves of small wavelengths.

[5] considered semi-analytical solutions for the Gray \& Scott model in a reaction-diffusion cell. The Galerkin method was used to obtain an ode model, as an approximation to the governing pdes system, and then the tools of combustion theory were used to analyze the ode model. Steady-state solutions and bifurcation diagrams were obtained and the region of parameter space, where Hopf bifurcations occur was also found. An excellent comparison was found between the results of the semi-analytical method and the numerical solutions of the governing pdes. Other papers to use this method of developing semi-analytical solutions include [6], who considered cubic-autocatalysis with Michaelis-Menten decay, and [7], who considered a class of generalized diffusive logistic delay equations.

Feedback control for chemical systems can stabilize both limit cycle solutions and unstable steady states and also generate chaotic behaviour. [8] considered numerical and experimental examination of the chlorine dioxide-iodine reaction in a CSTR, with separate feedback regulation of the input flow rate for the two different chemical species. They found that feedback control can lead to bursting, phenomena in which oscillations and uniform solutions alternate in time. [9] performed theoretical and numerical examination of temperature feedback control for an exothermic chemical reaction, with the rate governed by the Arrhenius law. They proved theorems related to the regions of parameter space free from oscillatory solutions and also applied appropriate restrictions to the gain, or feedback parameter, in order to avoid oscillatory solutions.

[10] considered a two-species chemotaxis model, comprising reaction-diffusion-advection equations, for bacteria density and a chemical attractant. The system exhibits complex pattern formation involving wave propagation and island formation. The cell is joined to a reservoir of chemoattractant, with the flux between the reservoir and the cell varied according to a control algorithm, in order to achieve a given bacteria profile. It was found that pattern formation could be suppressed and steady-state profiles achieved by this algorithm. [11] considered the FitzHugh-Nagumo model with global feedback delay. This system is bistable and the stationary front solution is subject to a bifurcation which destabilizes it. It is found that the feedback control can alter the velocity and the bifurcation point at which the instability occurs. [12] considered global feedback for the Gray \& Scott model to control spatiotemporal chaos. It was found that different control regimes could stabilize uniform steady-states or generate bistability between the uniform state and a travelling wave. 
In this paper, the reversible Selkov model (1) with feedback delay at the boundary is examined in a 1-D reaction-diffusion cell where the Galerkin method is used to develop semianalytical solutions. In $\S 2$ governing equations are presented and the Galerkin method is used to obtain the odes which represent the semi-analytical model. In $\S 3$ the steady-state concentration profiles and bifurcation patterns are presented and described in detail. In $\S 4$ a local stability analysis of the semi-analytical model is performed. The Hopf points are found for both feedback with and without delay; hence the parameter region in which Hopf bifurcations occur is identified and the effect of feedback strength and delay are explored. Comparisons are made throughout the paper between the semi-analytical results and numerical solutions of the governing pdes.

\section{The semi-analytical model}

\subsection{The governing equations}

The reversible Selkov model, or glycolytic oscillations model (1) is considered in a 1-D reaction-diffusion cell. The governing pdes and boundary conditions are

$$
\begin{aligned}
& s_{t}=D_{1} s_{x x}-\kappa s-s p^{2}+K_{2} p^{3}, \quad p_{t}=D_{2} p_{x x}-p+s p^{2}-K_{2} p^{3}, \\
& s_{x}=p_{x}=0, \text { at } x=0 \quad s=s_{0}, \quad p=p_{0}, \text { at } x=1 \text { and } t=0 .
\end{aligned}
$$

The system (2) is in non-dimensional form with the scaled concentrations of the reactant, $\mathrm{s}$ and autocatalyst, p. It is an open system; the reactor has a permeable boundary at $x=1$, joined to a reservoir which contains $\mathrm{s}$ and $\mathrm{p}$ at specified concentrations, $s_{0}$ and $p_{0}$, respectively. The boundary condition at $x=0$ is a zero-flux condition. The system has four other parameters; $\kappa$ and $K_{2}$ represent the rates of the reversible reactions in the first and second steps of (1). The parameter $K_{2}$ is relatively uninteresting as it does not alter the qualitative behavior of the system; we let $K_{2}=1$. We also assume that the decay of the precursor chemical $A \rightarrow S$ and reversible reaction of the final product $B \rightarrow P$ are both very small and can be neglected. The parameters $D_{1}$ and $D_{2}$ are the diffusion coefficients of the two species $s$ and $p$. This system has an unique steady-state solution and we let $\left(s_{s}, p_{s}\right)$ be the steady-state concentrations at $x=0$.

We are interested in examining the effect of feedback on the reaction-diffusion cell (2) and (3), so consider the following feedback algorithm

$$
s_{x}=p_{x}=0, \text { at } x=0, s=s_{0}+H\left(s(0, t-\tau)-s_{s}\right), \quad p=p_{0}+H\left(p(0, t-\tau)-p_{s}\right) \text { at } x=1,
$$

where the reservoir concentrations are altered, in response to the concentrations in the cell at the impermeable boundary located at $x=0$. Many studies have been undertaken of feedback control for CSTR's where the flow rate is altered in response to the concentrations in the reactor; (4) represents an analogous form of feedback control for a reaction-diffusion cell. This control mechanism could be implemented experimentally by measuring the concentrations at the non-permeable boundary $(x=0)$ and then altering the reservoir concentrations in response to those values. The feedback is proportional to the difference between the transient concentrations and the steady-state value at $x=0$, while $H$ is the strength of the feedback and $\tau$ is the delay response. The feedback (4) does not change the steady-state solutions of (3) so we investigate the effect of this feedback control on the stability of the reaction-diffusion cell. 
Numerical solutions of (2) are found using an explicit finite-difference scheme with accuracy of $O\left(\triangle t, \triangle x^{2}\right)$.

\subsection{The Galerkin method}

The Galerkin method assumes a spatial structure of the concentration profiles, which then allows the governing pdes (2) and boundary conditions (4) to be approximated by a set of lower-order odes. The expansion

$$
\begin{aligned}
s(x, t) & =s_{0}+H\left(s_{1}(t-\tau)+s_{2}(t-\tau)-s_{1 s}-s_{2 s}\right)+s_{2}(t) \cos \left(\frac{3}{2} \pi x\right) \\
& +\left(s_{1}(t)-H\left(s_{2}(t-\tau)+s_{1}(t-\tau)-s_{2 s}-s_{1 s}\right)\right) \cos \left(\frac{1}{2} \pi x\right), \\
p(x, t) & =p_{0}+H\left(p_{1}(t-\tau)+p_{2}(t-\tau)-p_{1 s}-p_{2 s}\right)+p_{2}(t) \cos \left(\frac{3}{2} \pi x\right) \\
& +\left(p_{1}(t)-H\left(p_{2}(t-\tau)+p_{1}(t-\tau)-p_{2 s}-p_{1 s}\right)\right) \cos \left(\frac{1}{2} \pi x\right),
\end{aligned}
$$

is used which represents a two-term method. Expansion (5) satisfies the boundary conditions (4), but not the governing pdes. The form of basis functions (5) also has the property that the concentrations at the impermeable boundary are $s=s_{0}+s_{1}+s_{2}$ and $p=p_{0}+p_{1}+p_{2}$, which do not explicitly depend on $H$. The steady-state concentrations are $s_{s}=s_{0}+s_{1 s}+s_{2 s}$ and $p_{s}=p_{0}+p_{1 s}+p_{2 s}$. Note that the form of (5) is not unique; a more symmetric form for could be chosen or quadratic expressions could be used, but the level of accuracy of the method is usually independent of the forms of the basis functions used. The free parameters in (5) are found by evaluating averaged versions of the governing equations, weighted by the basis functions. This procedure gives the odes

$$
\begin{aligned}
\frac{d s_{1}}{d t} & =-\frac{4 \kappa s_{0}}{\pi}-\frac{4 s_{0} p_{0}^{2}}{\pi}+\frac{4 K_{2} p_{0}^{3}}{\pi}-s_{1} p_{0}^{2}-\frac{3 s_{1} p_{1}^{2}}{4}+\frac{3 K_{2} p_{1}^{3}}{4}-\kappa s_{1}-\frac{s_{2} p_{1}^{2}}{4}-\frac{s_{1} p_{2}^{2}}{2} \\
& -\frac{s_{1} p_{1} p_{2}}{2}+\frac{3 K_{2} p_{1} p_{2}^{2}}{2}+\frac{3 K_{2} p_{1}^{2} p_{2}}{4}-2 s_{0} p_{0} p_{1}-\frac{D_{1} s_{1} \pi^{2}}{4}+3 K_{2} p_{0}^{2} p_{1}-\frac{72 s_{0} p_{2}^{2}}{35 \pi} \\
& -\frac{8 s_{0} p_{1}^{2}}{3 \pi}-s_{2} p_{1} p_{2}+\frac{16 K_{2} p_{0} p_{1} p_{2}}{5 \pi}-\frac{16 s_{0} p_{1} p_{2}}{15 \pi}-\frac{16 s_{1} p_{0} p_{2}}{15 \pi}-\frac{16 s_{2} p_{0} p_{1}}{15 \pi} \\
& +\frac{8 K_{2} p_{0} p_{1}^{2}}{\pi}-\frac{16 s_{1} p_{0} p_{1}}{3 \pi}-\frac{144 s_{2} p_{0} p_{2}}{35 \pi}+\frac{216 K_{2} p_{0} p_{2}^{2}}{35 \pi} \cdot \\
\frac{d p_{1}}{d t} & =-p_{1}-\frac{4 p_{0}}{\pi}+\frac{4 s_{0} p_{0}^{2}}{\pi}-\frac{4 K_{2} p_{0}^{3}}{\pi}+s_{1} p_{0}^{2}+\frac{3 s_{1} p_{1}^{2}}{4}-\frac{3 K_{2} p_{1}^{3}}{4}+\frac{s_{2} p_{1}^{2}}{4}+\frac{s_{1} p_{2}^{2}}{2}-\frac{D_{2} p_{1} \pi^{2}}{4} \\
& +\frac{s_{1} p_{1} p_{2}}{2}-\frac{3 K_{2} p_{1} p_{2}^{2}}{2}-\frac{3 K_{2} p_{1}^{2} p_{2}}{4}+2 s_{0} p_{0} p_{1}-3 K_{2} p_{0}^{2} p_{1}+\frac{72 s_{0} p_{2}^{2}}{35 \pi}+\frac{8 s_{0} p_{1}^{2}}{3 \pi}+s_{2} p_{1} p_{2} \\
& -\frac{16 K_{2} p_{0} p_{1} p_{2}}{5 \pi}+\frac{16 s_{0} p_{1} p_{2}}{15 \pi}+\frac{16 s_{1} p_{0} p_{2}}{15 \pi}+\frac{16 s_{2} p_{0} p_{1}}{15 \pi}-\frac{8 K_{2} p_{0} p_{1}^{2}}{\pi}+\frac{144 s_{2} p_{0} p_{2}}{35 \pi} \\
& -\frac{216 K_{2} p_{0} p_{2}^{2}}{35 \pi} . \\
\frac{d s_{2}}{d t} & =\frac{4 \kappa s_{0}}{3 \pi}-\kappa s_{2}-\frac{9 D_{1} s_{2} \pi^{2}}{4}+\frac{4 s_{0} p_{0}^{2}}{3 \pi}-\frac{4 K_{2} p_{0}^{3}}{3 \pi}-\frac{3 s_{2} p_{2}^{2}}{4}-s_{2} p_{0}^{2}+\frac{3 K_{2} p_{2}^{3}}{4}-\frac{s_{1} p_{1}^{2}}{4} \\
& +\frac{K_{2} p_{1}^{3}}{4}-\frac{s_{2} p_{1}^{2}}{2}+3 \kappa p_{0}^{2} p_{2}-2 s_{0} p_{0} p_{2}-s_{1} p_{1} p_{2}+\frac{3 K_{2} p_{1}^{2} p_{2}}{2}+\frac{8 s_{0} p_{2}^{2}}{9 \pi}-\frac{8 s_{0} p_{1}^{2}}{15 \pi}
\end{aligned}
$$




$$
\begin{aligned}
& +\frac{432 K_{2} p_{0} p_{1} p_{2}}{35 \pi}-\frac{144 s_{0} p_{1} p_{2}}{35 \pi}-\frac{144 s_{1} p_{0} p_{2}}{35 \pi}-\frac{144 s_{2} p_{0} p_{2}}{35 \pi}+\frac{8 K_{2} p_{0} p_{1}^{2}}{5 \pi}-\frac{16 s_{1} p_{0} p_{1}}{15 \pi} \\
& +\frac{16 s_{2} p_{0} p_{2}}{9 \pi}-\frac{8 K_{2} p_{0} p_{2}^{2}}{3 \pi} . \\
\frac{d p_{2}}{d t} & =\frac{4 p_{0}}{3 \pi}-p_{2}-\frac{9 D_{2} p_{2} \pi^{2}}{4}-\frac{4 s_{0} p_{0}^{2}}{3 \pi}+\frac{4 K_{2} p_{0}^{3}}{3 \pi}+\frac{3 s_{2} p_{2}^{2}}{4}+s_{2} p_{0}^{2}-\frac{3 K_{2} p_{2}^{3}}{4}+\frac{s_{1} p_{1}^{2}}{4} \\
& -\frac{K_{2} p_{1}^{3}}{4}+\frac{s_{2} p_{1}^{2}}{2}-3 \kappa p_{0}^{2} p_{2}+2 s_{0} p_{0} p_{2}+s_{1} p_{1} p_{2}-\frac{3 K_{2} p_{1}^{2} p_{2}}{2}-\frac{8 s_{0} p_{2}^{2}}{9 \pi}+\frac{8 s_{0} p_{1}^{2}}{15 \pi} \\
& -\frac{432 K_{2} p_{0} p_{1} p_{2}}{35 \pi}+\frac{144 s_{0} p_{1} p_{2}}{35 \pi}+\frac{144 s_{1} p_{0} p_{2}}{35 \pi}+\frac{144 s_{2} p_{0} p_{2}}{35 \pi}-\frac{8 K_{2} p_{0} p_{1}^{2}}{5 \pi}+\frac{16 s_{1} p_{0} p_{1}}{15 \pi} \\
& -\frac{16 s_{2} p_{0} p_{2}}{9 \pi}+\frac{8 K_{2} p_{0} p_{2}^{2}}{3 \pi} .
\end{aligned}
$$

The odes $(6)$ are for the case of no feedback $(H=0)$ as the terms associated with the feedback are excessively long to be presented here. It is found that a two-term method gives sufficient accuracy without excessive expression swell. The one-term solution (when $s_{2}=p_{2}=0$ ) is also calculated, for accuracy comparison purposes.

\section{Steady-state solutions}

The steady-state of (6) where $\frac{d s_{1}}{d t}=\frac{d s_{2}}{d t}=\frac{d p_{1}}{d t}=\frac{d p_{2}}{d t}=0$ represents a set of four transcendental equations $\left(f_{i}=0, i=1 \ldots 4\right)$, which are solved numerically using Maple. For the one term case, $s_{2}=p_{2}=0$, so in this case two transcendental equations are obtained.

Figures 1(a) and 1(b) show steady-state concentration profiles of $s$ and $p$ versus $x$, respectively. The parameters are $s_{0}=4, \kappa=0.01, D_{1}=D_{2}=0.08, H=0$ and $p_{0}=0.4$. The one and two-term semi-analytical and numerical solutions of (2) and (3) are shown. For this choice of parameters reactant and autocatalyst are both consumed in the reactor with a trough occurring at $x=0$. It can be clearly seen that the two-term expression gives a good approximation when compared with the numerical solution of the governing pdes. At $x=0$, the errors are less than $1 \%$ for the $s$ and $p$ concentrations. For the one-term approximation, the errors are slightly larger, but no greater than about 5\%. The two-term solutions are superior to the one-term profile as they model flat $s$-concentration profile more accurately.

Figures 2(a) and 2(b) show steady-state bifurcation diagrams, $s$ and $p$ versus $\kappa$, respectively. The concentrations are shown at $x=0$. This is an unique bifurcation pattern, with the steady state concentrations decreasing as $\kappa$ increases. The two-term solution is very close to the numerical solution of the pdes. The error for the one-term solution becomes very large as $\kappa$ increases and the solution becomes negative (unphysical) at $\kappa=0.68$. This is due to the one-term solution not approximating flat concentration profiles very well, as $\kappa$ becomes large. At $\kappa=0.6$ the numerical solutions are $(s, p)=(0.51,0.031)$ while the two-term semianalytical solutions are $(s, p)=(0.59,0.035)$. The errors between numerical and two-term semi-analytical solutions are $16 \%$ and $13 \%$ for the $s$ and $p$ concentrations, at $\kappa=0.6$.

\section{Local stability and oscillatory solutions}

Limit cycles and Hopf bifurcations points are known to occur for the system (1), in the CSTR. The theory of Hopf bifurcations is explained in standard texts on bifurcation theory 
and dynamical systems such as [13] or [14]. Here the stability of the semi-analytical model is analyzed and used to explore the effects of feedback (4) in altering the stability of the system (2) and (3). The Hopf degeneracy points are calculated to find a semi-analytical map in which Hopf bifurcations occur and this prediction is compared with numerical results. Hopf points are obtained by expanding in a Taylor series about the steady-state solution,

$$
\begin{array}{ll}
s_{1}(t)=s_{1 s}+\epsilon g e^{-\lambda t}, & p_{1}(t)=p_{1 s}+\epsilon g_{1} e^{-\lambda t}, \\
s_{2}(t)=s_{2 s}+\epsilon h e^{-\lambda t}, & p_{2}(t)=p_{2 s}+\epsilon h_{1} e^{-\lambda t} .
\end{array}
$$

We then substitute (7) into the odes (6), and linearize around the steady state. The eigenvalues, $\lambda$, of the Jacobian matrix are found by solving the characteristic equation and describe the evolution of small perturbations to the steady-state solution. For the case in which there is no feedback delay, $\tau=0$, the characteristic equation is given by the equation,

$$
\lambda^{4}+\alpha_{1} \lambda^{3}+\alpha_{2} \lambda^{2}+\alpha_{3} \lambda+\alpha_{4}=0 .
$$

Hopf bifurcations occur for this system when one pair of eigenvalues is purely imaginary, which implies

$$
q=\alpha_{4} \alpha_{1}^{2}+\alpha_{3}^{2}-\alpha_{1} \alpha_{2} \alpha_{3}=0 .
$$

Hence the degenerate Hopf points are given by

$$
f_{i}=q=\frac{d q}{d \kappa}=0, \quad i=1 \ldots 4 .
$$

The Hopf bifurcation points are found by solving (10), which gives curves in the $s_{0}-p_{0}$ and $D_{1}-D_{2}$ planes. Here, the degenerate curves represent both physical and non-physical solutions (where the rate constant $\kappa$ is negative). To obtain physically realistic Hopf bifurcations (where $\kappa>0$ ) we replace the condition $\frac{d q}{d \kappa}=0$ by $\kappa=0$. Hence we obtain

$$
f_{i}=q=\kappa=0, \quad i=1 \ldots 4,
$$

as an alternative condition to (10) for physically realistic Hopf points.

For the case in which the feedback delay $\tau \neq 0$, the characteristic equation has a nonpolynomial form, so we set $\lambda=i \omega$ in the characteristic equation and separate into real, $q_{1}$, and imaginary, $q_{2}$, parts. The Hopf bifurcation points occur at points where $\lambda$ is purely imaginary, for more details see [15] and [16]. The Hopf bifurcation points are found by solving the system of equations plus $\kappa=0$ which gives

$$
f_{1}=f_{2}=f_{3}=f_{4}=q_{1}=q_{2}=\kappa=0,
$$

as an generalisation of (11), for the case where the feedback delay is non-zero. Note that (11) and (12) are equivalent for the case of $\tau=0$. Also note that due to the characteristic equation being of a non-polynomial form when the feedback delay is non-zero, expressions for degenerate Hopf points (the equivalent of (10)) are not derived.

Figure 3 shows the regions in the $s_{0}-p_{0}$ plane in which Hopf bifurcation occur. Shown are the solutions to both (10) and (11) plus numerical solutions. The other parameters are $D_{1}=D_{2}=0.08, H=0$ and $\tau=0$. The degenerate curve, found by solving (10), breaks the plane into upper and lower regions. Above this curve only stable solutions occur and there are no Hopf bifurcation points while under the curve Hopf bifurcation points occur, both 
for physical and non-physical solutions. In addition, the looped curve encloses the region in which physically realistic Hopf bifurcation points occur, which are given by solving the conditions (11), so $\kappa \geq 0$. For the looped region the maximum numerical values at which Hopf bifurcations can occur are $s_{0}=5.60, p_{0}=0.469$ while the two-term semi-analytical maximum values are $s_{0}=5.67$ and $p_{0}=0.476$. Also, the numerical region has minimum values of $s_{0}=4.35$ and $p_{0}=0.292$ and $s_{0}=4.33$ and $p_{0}=0.280$, for two-term semianalytical solutions. The errors in these estimates are less than $4 \%$. It can be clearly seen that the two-term expression gives an excellent estimate of the region in which physically realistic Hopf points occur.

Figure 4 shows regions in the $D_{1}-D_{2}$ plane in which Hopf bifurcation occur. Shown are the solutions to both (10) and (11) and numerical solutions. The other parameters are $s_{0}=5, p_{0}=0.4, H=0$ and $\tau=0$. This figure represents an alternative view of the Hopf bifurcation parameter space, compared that shown in figure 3 . The curve, which cuts the plane into two, was generated by solving the conditions (10), describes the full set of degenerate Hopf bifurcation points. Above this curve only stable solutions occur and there are no Hopf bifurcation points while under the curve Hopf bifurcation points occur. Again, inside the looped curve physically realistic Hopf bifurcation points occur, which are given by solving the conditions (11). For the looped region, the maximum numerical values are $D_{1}=0.092$ and $D_{2}=0.150$ while the two-term semi-analytical maximum values are $D_{1}=0.086$ and $D_{2}=0.146$. The errors in these estimates are less than $7 \%$ and $3 \%$, for the value of $D_{1}$ and $D_{2}$ respectively. Furthermore, the minimum two-term semi-analytical values are $D_{1}=0.036$ and $D_{2}=0.019$, with minimum numerical values of $D_{1}=0.030$ and $D_{2}=0.030$. In this region of the figure, the relative errors are quite large but the absolute errors are still quite low, no greater than 0.011. Overall the semi-analytical looped region is a good approximation to the numerically obtained regions.

Figure 5 shows the regions of the $s_{0}-p_{0}$ plane in which Hopf bifurcations can occur, for different values of the feedback strength $H=0,0.1$ and 0.2 . The other parameters are $D_{1}=D_{2}=0.08$ and $\tau=0$. Increasing the feedback strength $H$ leads to the degenerate curve moving upwards and the looped region, in which physically realistic Hopf bifurcations occur also expands. For example, the point $\left(s_{0}, p_{0}\right)=(4.4,0.49)$ is stable when $H=0$ but, it is unstable for both $H=0.1$ and 0.2 while the point $\left(s_{0}, p_{0}\right)=(4.3,0.53)$ is stable for both $H=0$ and 0.1 but is unstable when $H=0.2$. Hence increasing the feedback strength $H$ causes the region of instability to increase in area. Figure 6 shows the regions of the $D_{1}-D_{2}$ plane in which Hopf bifurcations occur, for $H=0,0.1$ and 0.2 . The other parameters are $s_{0}=5$, $p_{0}=0.4$ and $\tau=0$. Increasing the feedback parameter $H$ causes the region of instability to increase in the $D_{1}-D_{2}$ plane, a similar effect to that which occurs in the $s_{0}-p_{0}$ plane. For example, the point $\left(D_{1}, D_{2}\right)=(0.09,0.2)$ is stable with $H=0$ but, it is unstable for both $H=0.1$ and $H=0.2$. Moreover, the point $\left(D_{1}, D_{2}\right)=(0.09,0.16)$ is stable for both $H=0$ and 0.1 while, it is unstable where $H=0.2$.

Figure 5 shows the regions of the $s_{0}-p_{0}$ plane in which Hopf bifurcations can occur, for different values of feedback strength $H=0,-0.1$ and -0.2 . The other parameters are $D_{1}=D_{2}=0.08$ and $\tau=0$. The other parameters are the same as for figure 5 but here the feedback strength $H$ is negative. Decreasing $H$, to negative values with larger magnitude, leads to the degenerate curve moving downwards and reductions in the area of the looped region in which physically realistic Hopf bifurcations occur. Figure 8 shows the regions of the $D_{1}-D_{2}$ plane in which Hopf bifurcations occur, for $H=0,-0.1$ and -0.2 . The other parameters are $s_{0}=5, p_{0}=0.4$ and $\tau=0$, the same as figure 6 . Here we see that decreasing 
the feedback parameter $H$ (to negative values with larger magnitude) leads to the looped region of instability decreasing in the $D_{1}-D_{2}$ plane. For example, the point $\left(D_{1}, D_{2}\right)=$ $(0.08,0.12)$ is unstable with $H=0$ but, it is stable for both $H=-0.1$ and -0.2 . Moreover, the point $\left(D_{1}, D_{2}\right)=(0.07,0.1)$ is unstable for both $H=0$ and -0.1 while, it is stable where $H=-0.2$. In summary, larger values of $H$ destabilize the system leading to growth in the region in which Hopf bifurcations occur while smaller values of $H$ stabilize the system, leading the enclosed looped region to shrink.

Now the effect of including delay in the feedback response is considered. Figure 9 shows the Hopf bifurcations regions in the $s_{0}-p_{0}$ plane. The delay $\tau=0,1$ and 2 while the other parameters are $D_{1}=D_{2}=0.08$ and $H=0.2$. Here, for $\tau \neq 0$ the solutions can be found by solving condition (12). The line, representing the full set of degenerate Hopf points, is missing for this figure as a theoretical expression to calculate it is unavailable. The figure shows that the looped Hopf region increases in size as the delay parameter increases. For example, the point $\left(s_{0}, p_{0}\right)=(4.1,0.54)$ is a stable point for $\tau=0$ but it is unstable for both $\tau=1$ and $\tau=2$. Also, the point $\left(s_{0}, p_{0}\right)=(3.9,0.55)$ is a stable point for both $\tau=0$ and $\tau=1$ while it is unstable for $\tau=2$. It can be seen that including information from the more distant past (larger values of $\tau$ ) destabilizes the system. Figure 10 shows that the Hopf bifurcation regions in the $D_{1}-D_{2}$ plane. The delay $\tau=0,1.5$ and 2.2 while the other parameters are $p_{0}=0.4$, $s_{0}=5$ and $H=0.2$. For $0<\tau<1.94$, the Hopf bifurcation region in the $D_{1}-D_{2}$ plane reduces in size as $\tau$ increases, stabilizing the system but for $\tau \geq 1.94$ the Hopf bifurcation region rebounds and expands in size. So the general trend is that larger values of the delay parameter $\tau$ destabilize the system but these results show that this is not always a monotonic effect.

Figure 11(a) shows a limit cycle solution in the $s$ versus $p$ phase plane, while 11(b) and 11(c) show the evolution of $s$ and $p$ versus $t$, respectively, at $x=0$. The parameters are $p_{0}=0.4, D_{1}=D_{2}=0.08, H=0.1, s_{0}=5, \kappa=0.003$ and $\tau=0$. Shown are the two-term semi-analytical solution and the numerical solution. For this choice of parameters a limit cycle occurs. The numerical period of the limit cycle of the reactant and autocatalyst is 18.78 while, the amplitudes of the limit cycle are 2.29 and 0.83 for the reactant and autocatalyst concentrations, respectively. The two-term semi-analytical period is 19.79 and the amplitudes are 2.37 and 0.81 for the reactant and autocatalyst concentrations, respectively. The errors in the two-term semi-analytical values, compared to numerical solutions of (2) and (4) are less than 5\%. Also the time evolution curves show that the locations of the first few peaks and troughs of the semi-analytical solutions are close to the numerical values.

Figure 12(a) and (b) shows the evolution of $s$ and $p$, versus $t$, at $x=0$. The parameters are $p_{0}=0.4, D_{1}=D_{2}=0.08, H=0.1, s_{0}=4$ and $\kappa=0.05$. Shown are two-term semi-analytical solution and the numerical solution. For the time becomes large, the solution evolves to a steady-state, with $s \simeq 3.22$ and $p \simeq 0.09$ for the two-term semi analytical solutions. For numerical solutions, the solution evolves to a steady-state, with $s \simeq 3.13$ and $p \simeq 0.10$. The errors between two-term semi-analytical solutions and numerical solutions are $3 \%$ and $10 \%$ for the $s$ and $p$ concentrations. It can be seen that the two-term semi-analytical solution gives a good approximation, when compared with the numerical solution of the governing pdes, of the steady-state values and of the relaxation oscillations. 


\section{Conclusion}

Semi-analytical solutions have been developed for the reversible Selkov model with feedback delay in a reaction-diffusion cell. Comparisons with numerical solutions show that the twoterm semi-analytical model is very accurate. The effect of feedback both with and without delay has been considered, on the regions in which Hopf bifurcations occur. It is shown that increasing the strength of the feedback destabilises the system while decreasing the strength, through negative values, stabilizes the system. The effect of delay in the feedback response is usually to destabilize the system but the results show this is not always a monotonic effect and that small delays can sometimes have a stabilizing effect. Future work will apply the method to a more complex version of the reversible Selkov model in a reaction-diffusion cell, which includes the effects of a precursor chemical.

\section{Acknowledgements}

The authors would like to thank an anonymous referee for their helpful comments. K.S. Al Noufaey gratefully thanks Taif University in Saudi Arabia for awarding him a PhD scholarship to study at the University of Wollongong, Australia.

\section{References}

[1] J. M. L. Corbel, J. N. J. Van Lingen, J. F. Zevenbergen, O. L. J. Gijzeman, A. Meijerink, Strobes: pyrotechnic compositions that show a curious oscillatory combustion, Angew. Chem. Int. Ed. 52 (2013) 290-303.

[2] P. Richter, P. Regmus, J. Ross, Control and dissipation in oscillatory chemical engines, Prog. Theor. Phys. 66 (1981) 385-405.

[3] E. E. Selkov, Self-oscillations in glycolysis, Eur. J. Biochem. 4 (1968) 79-86.

[4] A. K. Dutt, The effect of diffusion on the Hopf-bifurcation in a model glycolytic reaction exhibiting oscillations, Chem. Phys. Lett. 357 (2002) 341-345.

[5] T. R. Marchant, Cubic autocatalytic reaction-diffusion equations: semi-analytical solutions, Proc. Roy. Soc. Lond A 458 (2002) 873-888.

[6] T. Marchant, Cubic autocatalysis with Michaelis-Menten kinetics: semi-analytical solutions for the reaction-diffusion cell, Chem. Engng. Sci. 59 (2004) 3433-3440.

[7] H. Alfifi, T. Marchant, M. Nelson, Generalised diffusive delay logistic equations: semianalytical solutions, Dyn. Contin. Discrete Ser. B. 19 (2012) 579-596.

[8] M. Dolnik, A. Banks, I. Epstein, Oscillatory chemical reaction in a CSTR with feedback control of flow rate, J. Phys. Chem. 101 (1997) 5148-5154.

[9] M. Sexton, L. Forbes, B. Gray, A study of the dynamics of a continuously stirred tank reactor with feedback control of the temperature, Dynam. Sys. 12 (1997) 89-108.

[10] U. Brant-Pollmann, D. Lebiedz, M. Diehl, S. Sager, J. Schloder, Real-time nonlinear feedback control of pattern formation in (bio)chemical reaction-diffusion processes: a model study, Chaos 15 (2005) 033901. 
[11] Y.-F. He, B.-Q. Ai, B. Hu, Pattern formation controlled by time delayed feedback in bistable media, J. Chem. Phys. 133 (2010) 114507.

[12] Y. Kyrychko, K. Blyuss, S. Hogan, E. Scholl, Control of spatiotemporal patterns in the Gray-Scott model, Chaos 19 (2009) 043126.

[13] J. Guckenheimer, P. Holmes, Nonlinear Oscillations, Dynamical systems, and Bifurcations of Vector Fields, Springer-Verlag, New York, 1983.

[14] M. Golubitsky, D. G. Schaeffer, Singularites and Groups in Bifurcation Theory, SpringerVerlag, New York, 1985.

[15] K. Miroslav, Delay Compensation for Nonlinear, Adaptive, and PDE Systems, Birkhauser Boston Inc, 2009.

[16] H. Alfifi, T. Marchant, M. Nelson, Semi-analytical solutions for the 1- and 2-D diffusive Nicholsons blowflies equation, IMA J. Appl. Math. (to appear, 2014). 


\section{Figure Captions}

Figure 1 Steady-state reactant $s$, (a), and autocatalyst $p,(\mathrm{~b})$, concentration profiles versus $x$. The parameters are $s_{0}=4, \kappa=0.01, D_{1}=D_{2}=0.08$ and $p_{0}=0.4$. The oneterm (black, dashed-dotted), two-term (blue, dashed) semi-analytical solution and the numerical solution of (2) and (3)(red, dotted) are shown.

Figure 2 Steady-state bifurcation diagrams, reactant $s$, (a), and autocatalyst $p$, (b), versus $\kappa$. The concentrations are shown at $x=0$. The parameters are $s_{0}=4, D_{1}=D_{2}=0.08$ and $p_{0}=0.4$. The one-term (green, solid), two-term (blue, dash-dotted) semi-analytical solution and the numerical solution of (2) and (3) (red, dotted) are shown.

Figure 3 The regions of the $s_{0}-p_{0}$ plane in which Hopf bifurcation can occur. The twoterm (blue, dashes) region and numerical solutions (red, squares) are shown. The other parameters are $D_{1}=D_{2}=0.08, H=0$ and $\tau=0$.

Figure 4 The regions of the $D_{1}-D_{2}$ plane in which Hopf bifurcation can occur. The twoterm (blue, dashes) region and numerical solutions (red, squares) are shown. The other parameters are $s_{0}=5, p_{0}=0.4, H=0$ and $\tau=0$.

Figure 5 Hopf bifurcation curves in the $s_{0}-p_{0}$ plane. Shown is the two-term semi-analytical solutions with feedback strength $H=0$ (black dash-dotted), $H=0.1$ (green, solid) and $H=0.2$ (red, dotted). The other parameters are $D_{1}=D_{2}=0.08$ and $\tau=0$.

Figure 6 Hopf bifurcation curves in the $D_{1}-D_{2}$ plane. Shown is the two-term semianalytical solutions with feedback strength $H=0$ (black dash-dotted), $H=0.1$ (green, short-dashed dotted) and $H=0.2$ (red, dotted). The other parameters are $s_{0}=5$, $p_{0}=0.4$ and $\tau=0$.

Figure 7 Hopf bifurcation curves in the $s_{0}-p_{0}$ plane. Shown is the two-term semi-analytical solutions with feedback strength $H=0$ (black, dash-dotted), $H=-0.1$ (green, solid) and $H=-0.2$ (red, dotted). The other parameters are $D_{1}=D_{2}=0.08$ and $\tau=0$.

Figure 8 Hopf bifurcation curves in the $D_{1}-D_{2}$ plane. Shown is the two-term semianalytical solutions with feedback strength $H=0$ (black dashed-dotted), $H=-0.1$ (green, short dash-dotted) and $H=-0.2$ (red, dotted). The other parameters are $s_{0}=5, p_{0}=0.4$ and $\tau=0$.

Figure 9 The regions Hopf bifurcations occur in the $s_{0}-p_{0}$ plane. The delay parameter $\tau=0$ (black, dash-dotted), $\tau=1$ (green, solid) and $\tau=2$ (red, dotted) while the other parameters are $D_{1}=D_{2}=0.08$ and $H=0.2$.

Figure 10 The regions Hopf bifurcations occur in the $D_{1}-D_{2}$ plane. The delay parameter $\tau=0$ (black dash-dotted), $\tau=1.5$ (green, dashes) and $\tau=2.2$ (red, dotted) while the other parameters are $s_{0}=5, p_{0}=0.4$ and $H=0.2$.

Figure 11 The limit cycle curve $s$ versus $p$, (a), and the evolution of $s$, (b), and $p$, (c), at $x=0$ versus $t$. The two-term semi-analytical solution (blue, dashes) and the numerical solution (red, dotted) are shown. The parameters are $p_{0}=0.4, D_{1}=D_{2}=0.08$, $H=0.1, s_{0}=5, \kappa=0.003$ and $\tau=0$. 
Figure 12 The evolution of $s$, (a), and $p$, (b), at $x=0$ versus $t$. The two-term semi-analytical solution (red, dotted) and the numerical solution (blue, dashes) are shown. The parameters are $p_{0}=0.4, D_{1}=D_{2}=0.08, H=0.1, s_{0}=4$ and $\kappa=0.05$. 


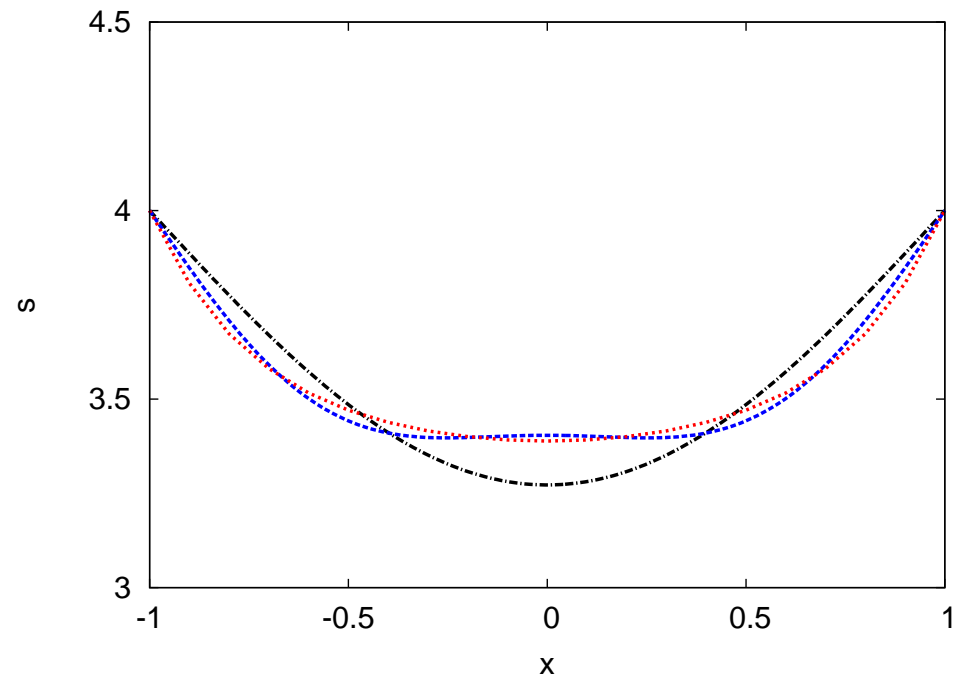

(a)

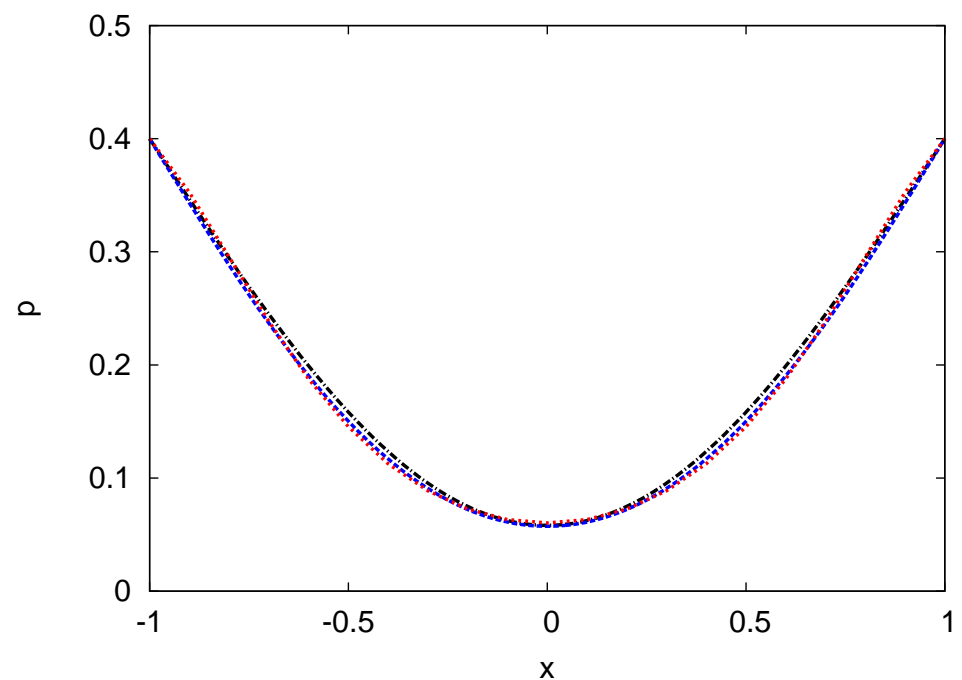

(b)

Figure 1: 


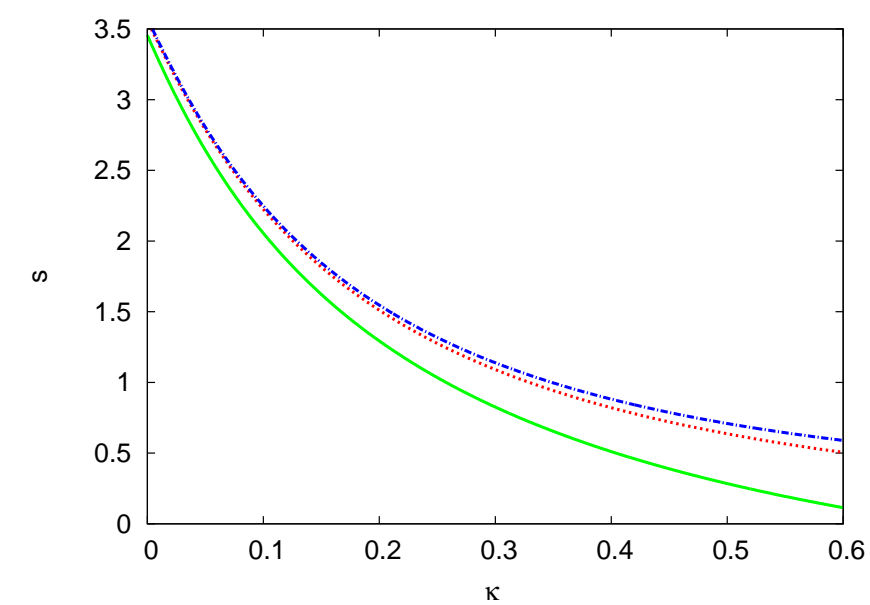

(a)

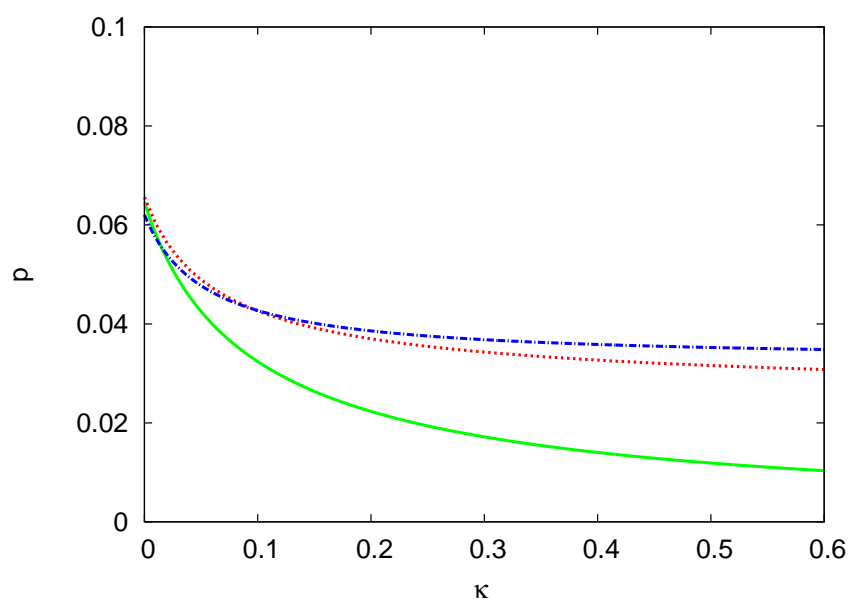

(b)

Figure 2: 


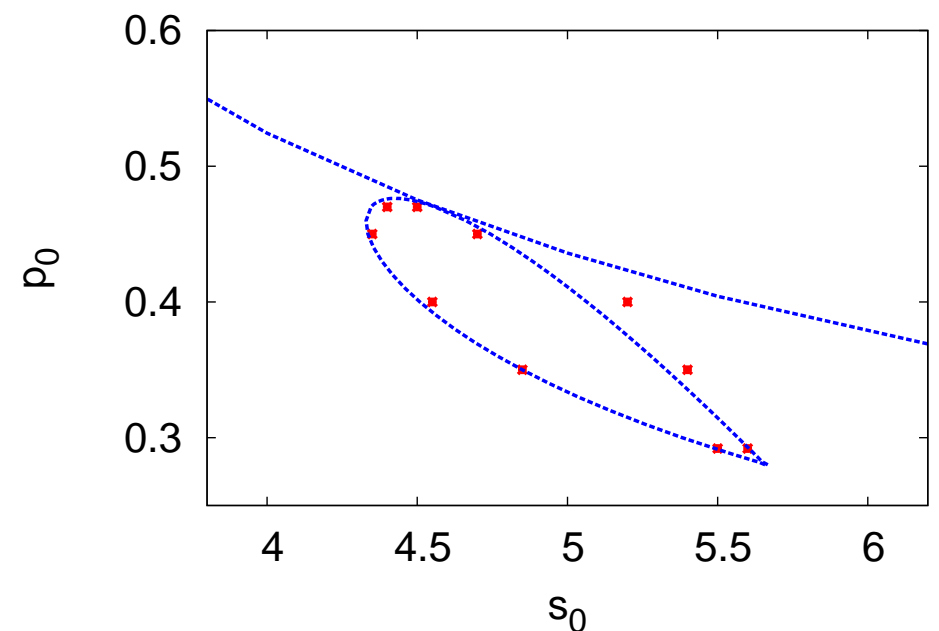

Figure 3:

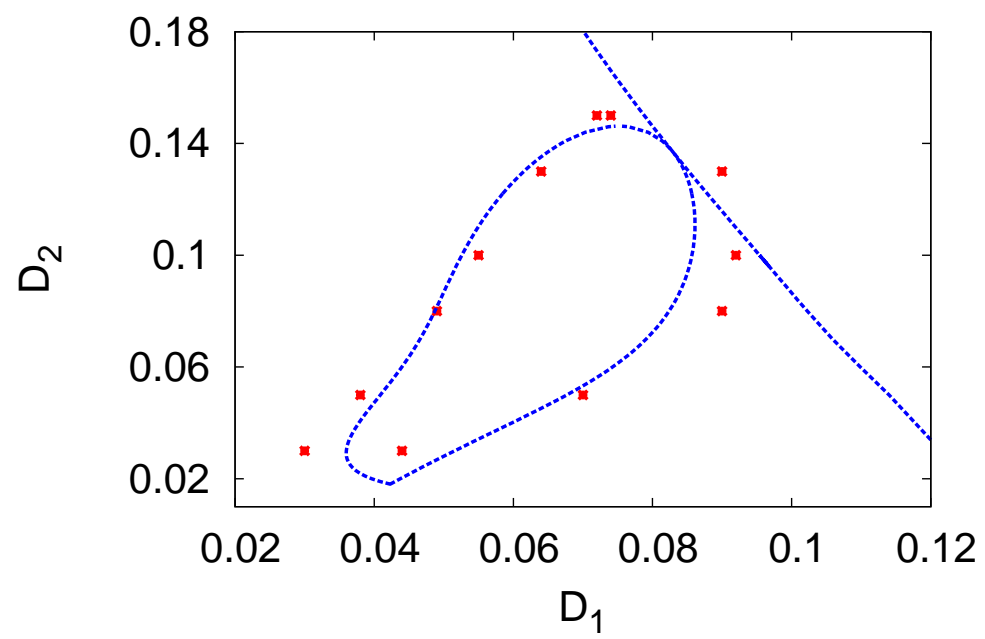

Figure 4: 


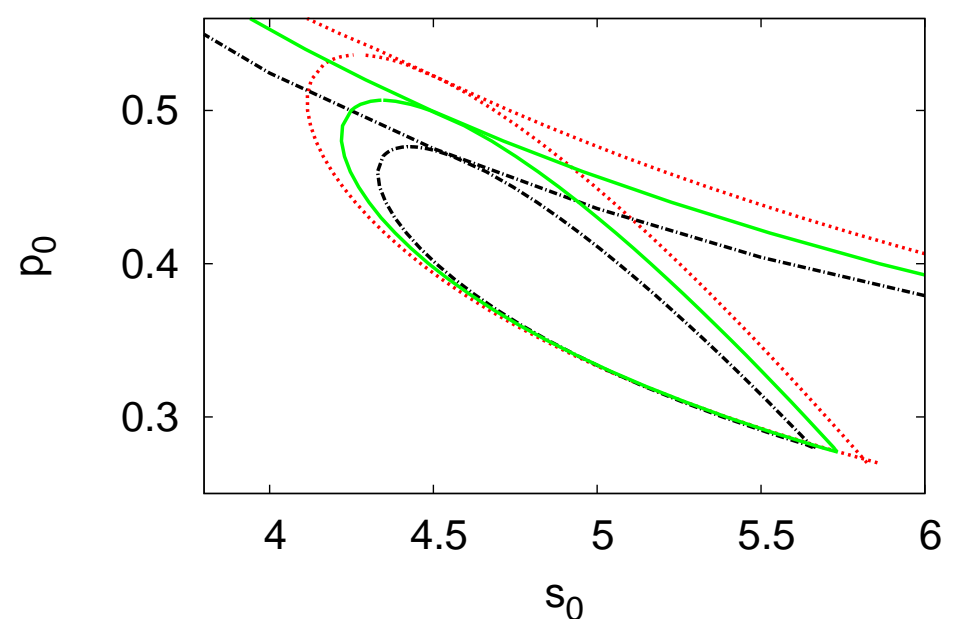

Figure 5:

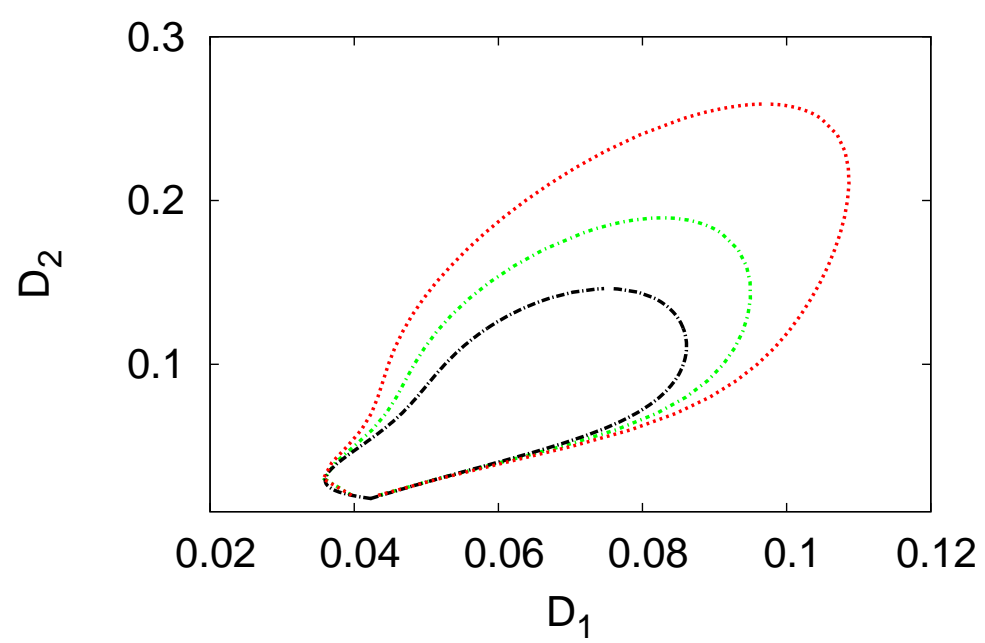

Figure 6: 


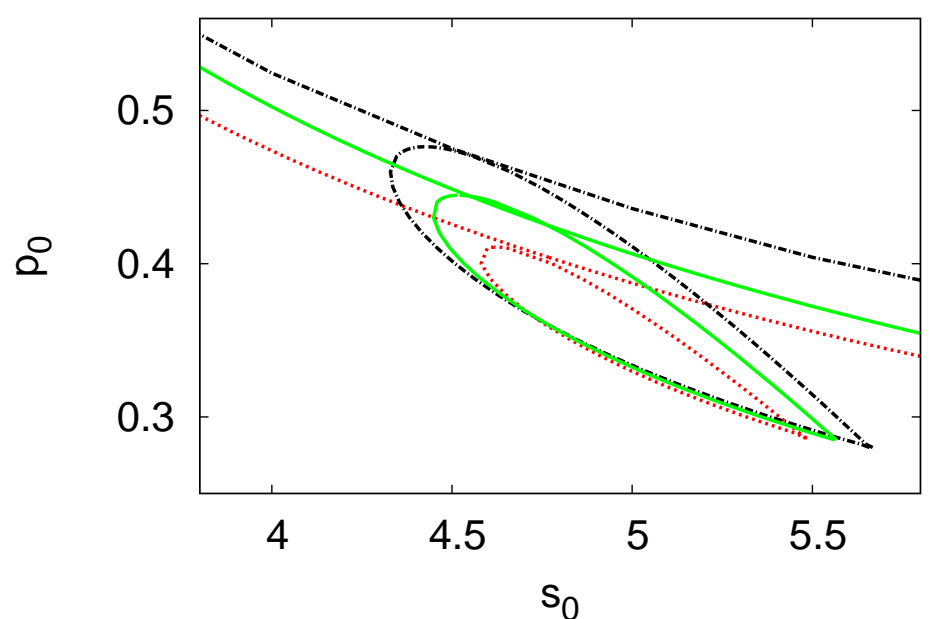

Figure 7:

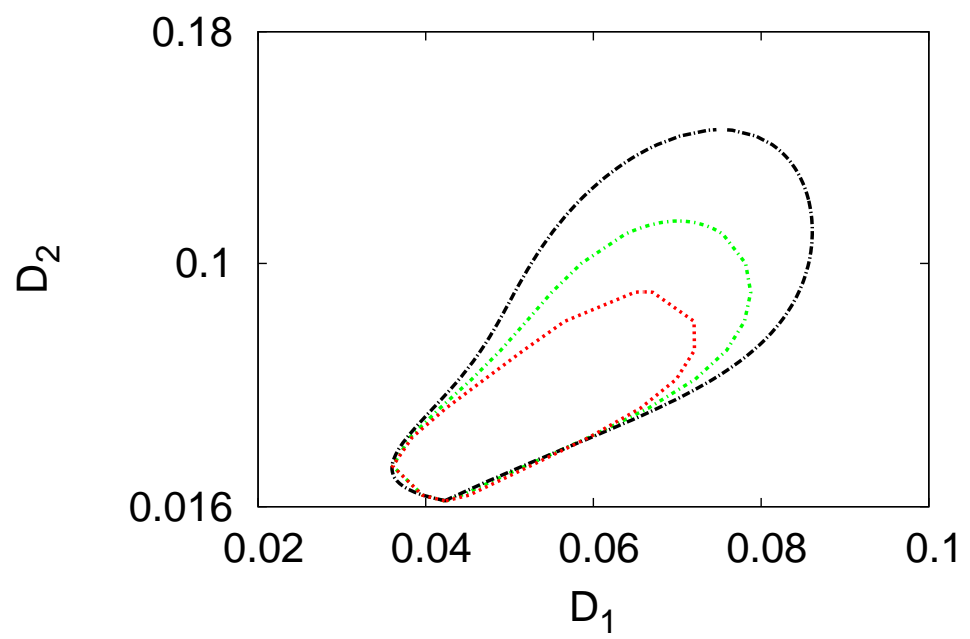

Figure 8: 


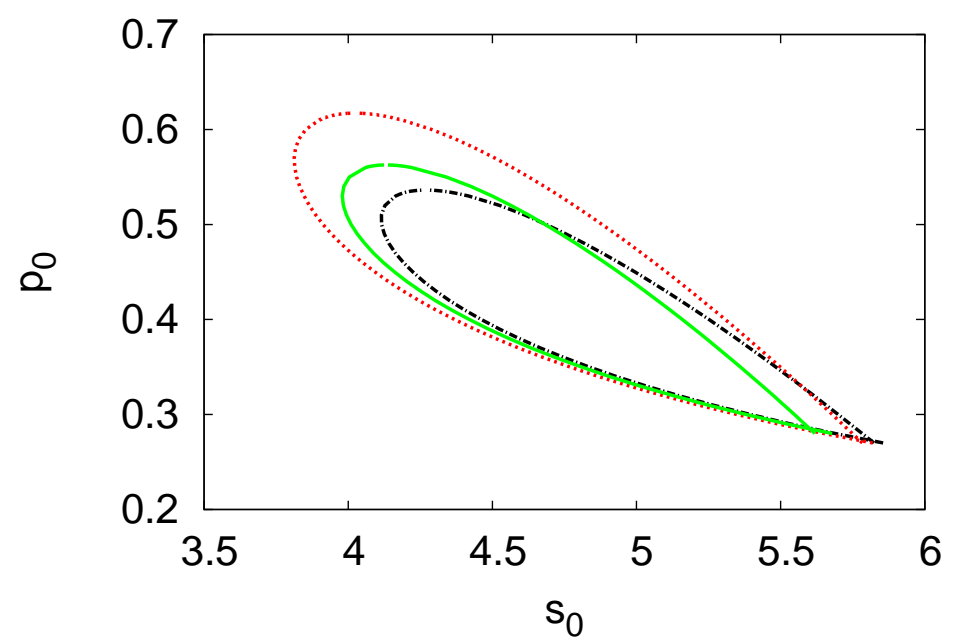

Figure 9:

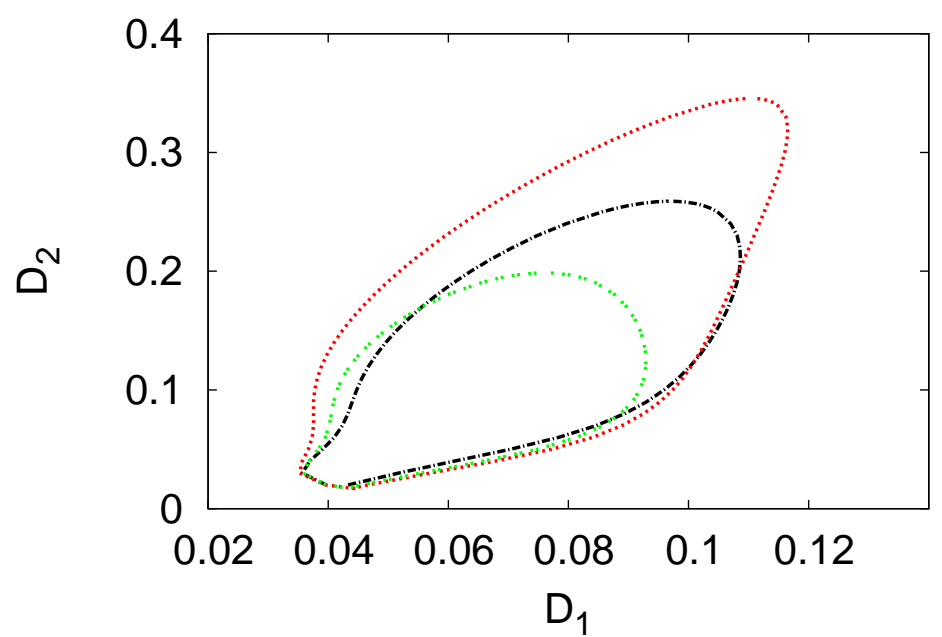

Figure 10: 


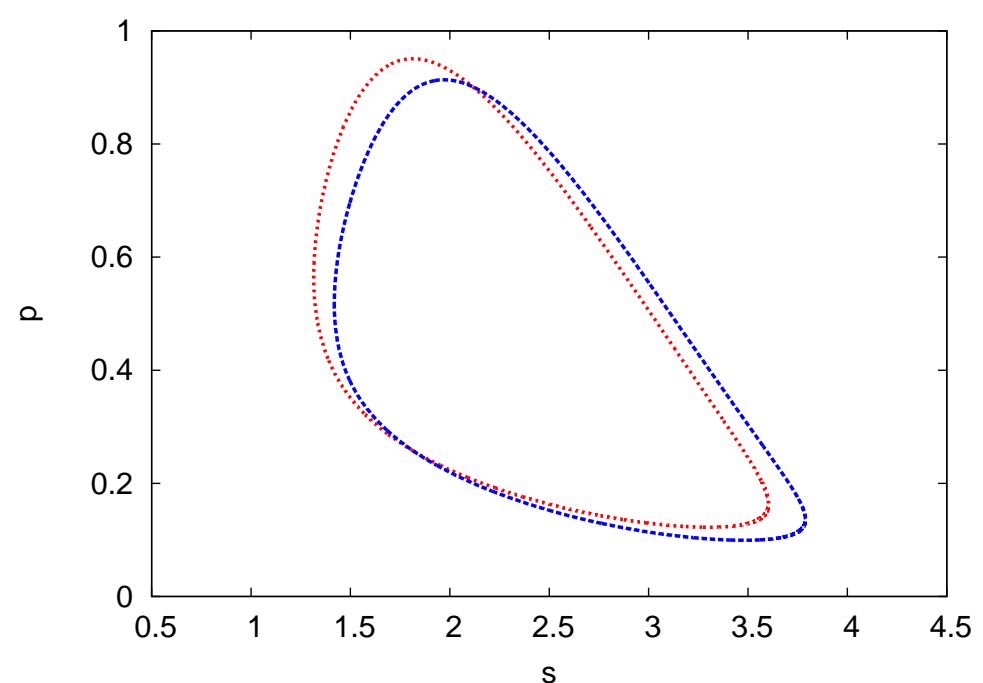

(a)

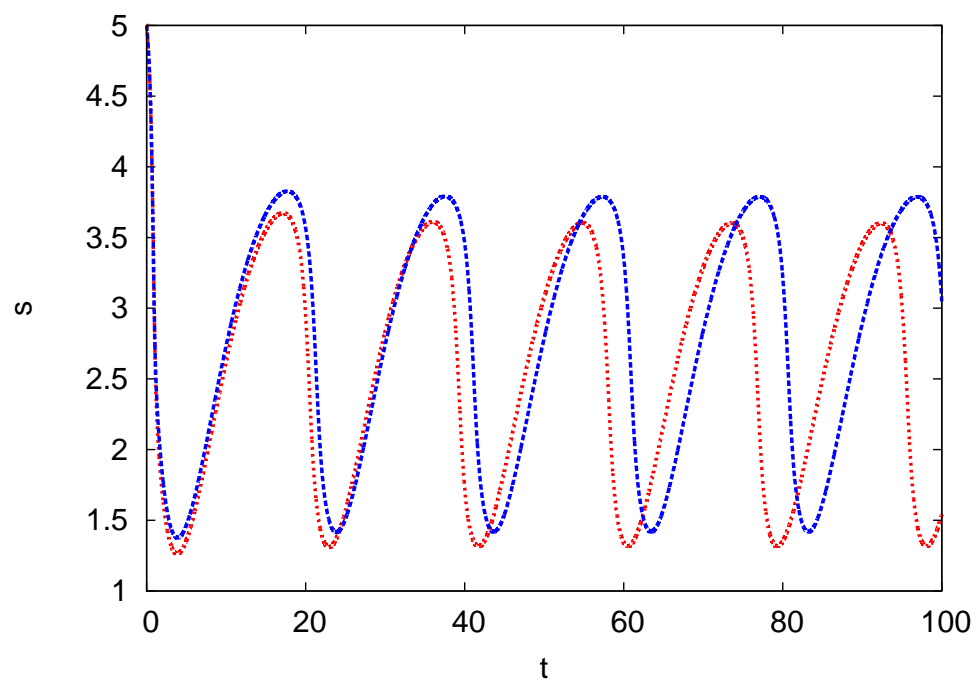

(b)

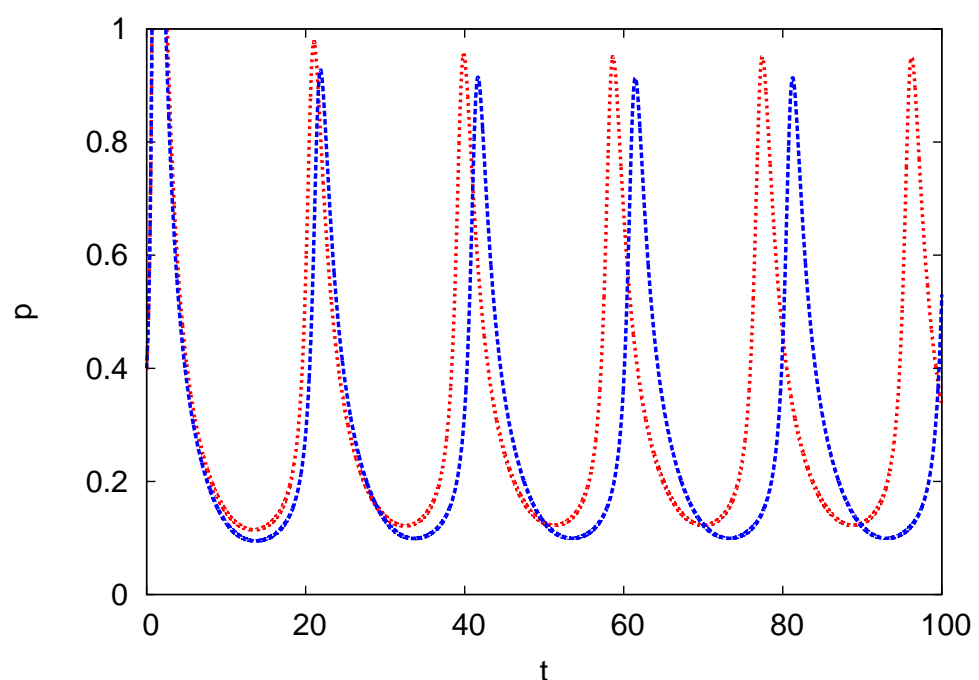

(c)

Figure 11: 


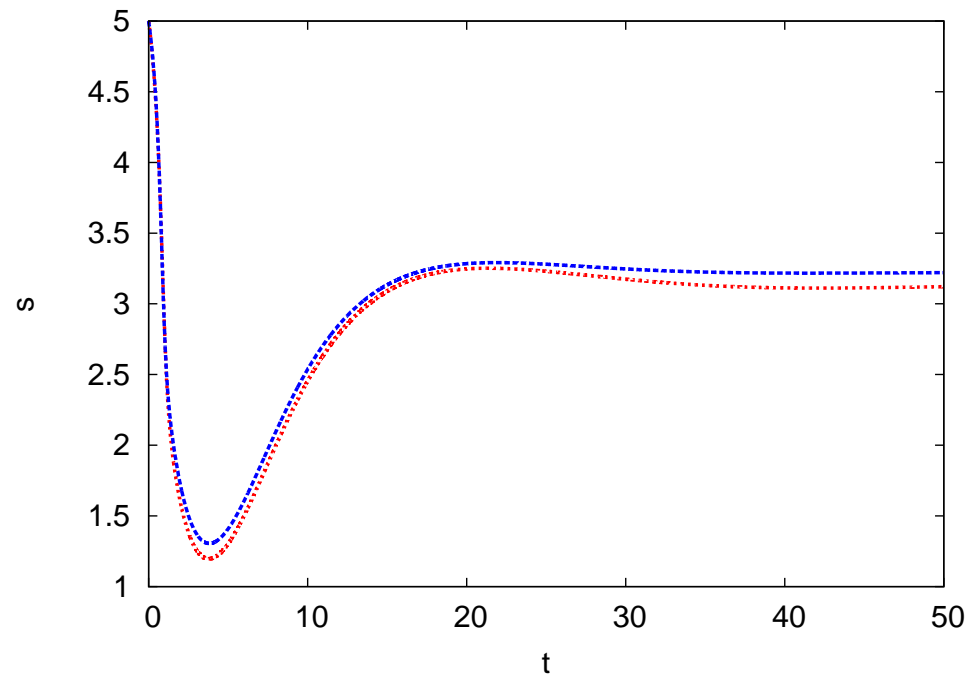

(a)

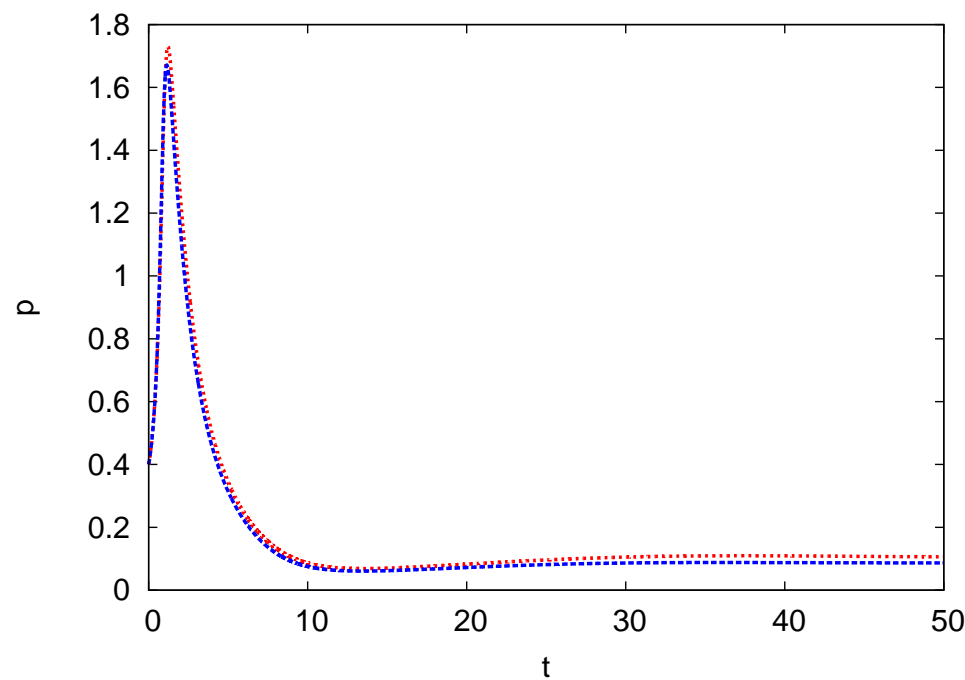

(b)

Figure 12: 